\title{
THE USE OF ADOMIAN DECOMPOSITION METHOD FOR SOLVING PROBLEMS IN CALCULUS OF VARIATIONS
}

\author{
MEHDI DEHGHAN AND MEHDI TATARI
}

Received 16 March 2005; Revised 9 August 2005; Accepted 12 September 2005

Dedication to Professor John C. Butcher (University of Auckland, New Zealand) on the occasion of his birthday

In this paper, a numerical method is presented for finding the solution of some variational problems. The main objective is to find the solution of an ordinary differential equation which arises from the variational problem. This work is done using Adomian decomposition method which is a powerful tool for solving large amount of problems. In this approach, the solution is found in the form of a convergent power series with easily computed components. To show the efficiency of the method, numerical results are presented.

Copyright (c) 2006 M. Dehghan and M. Tatari. This is an open access article distributed under the Creative Commons Attribution License, which permits unrestricted use, distribution, and reproduction in any medium, provided the original work is properly cited.

\section{Introduction}

In the large number of problems arising in analysis, mechanics, geometry, and so forth, it is necessary to determine the maximal and minimal of a certain functional. Because of the important role of this subject in science and engineering, considerable attention has been received on this kind of problems. Such problems are called variational problems.

There are three problems that have an important role in the development of the calculus of variations [16].

The problem of brachistochrone is proposed in 1696 by Johann Bernoulli which is required to find the line connecting two certain points $A$ and $B$ that do not lie on a vectorial line and possessing the property that a moving particle slides down this line from $A$ to $B$ in the shortest time. This problem was solved by Johann Bernoulli, Jacob Bernoulli, Leibnitz, Newton, and L'Hospital. It is shown that the solution of this problem is a cycloid.

In the problem of geodesics we want to determine the line of minimum length connecting two given points on a certain surface. This problem was solved in 1698 by Jacob Bernoulli and a general method for solving such problems was given in the works of Euler and Lagrange. 
In the isoperimetric problem, it is required to find a closed line of given length $l$ bounding a maximum area $S$. The solution of this problem is circle. General methods for solving problems with isoperimetric conditions were elaborated by Euler.

More historical comments about variational problems are found in [16, 17].

The simplest form of a variational problem can be considered as

$$
v[y(x)]=\int_{x_{0}}^{x_{1}} F\left(x, y(x), y^{\prime}(x)\right) d x,
$$

where $v$ is the functional that its extremum must be found. To find the extreme value of $v$, the boundary points of the admissible curves are known in the following form:

$$
y\left(x_{0}\right)=\alpha, \quad y\left(x_{1}\right)=\beta .
$$

One of the popular methods for solving variational problems are direct methods. In these methods the variational problem is regarded as a limiting case of a finite number of variables. This extremum problem of a function of a finite number of variables is solved by ordinary methods, then a passage of limit yields the solution of the appropriate variational problem [16]. The direct method of Ritz and Galerkin has been investigated for solving variational problems in $[16,17]$. Using Walsh series method, a piecewise constant solution is obtained for variational methods [10]. Some orthogonal polynomials are applied on variational problems to find continuous solutions for these problems $[9,18,19]$. Also Fourier series and Taylor series are applied to variational problems, respectively in $[21,22]$, to find a continuous solution for this kind of problems.

The necessary condition for the solution of the problem (1.1) is to satisfy the EulerLagrange equation

$$
F_{y}-\frac{d}{d x} F_{y^{\prime}}=0
$$

with the boundary conditions given in (1.2). The boundary value problem (1.3) does not always have a solution and if the solution exists, it may not be unique. Note that in many variational problems the existence of a solution is obvious from the physical or geometrical meaning of the problem, and if the solution of Euler's equation satisfies the boundary conditions, it is unique, then this unique extremal will be the solution of the given variational problem [16]. Thus another approach for solving variational problem (1.1) is finding the solution of the ordinary differential equation (1.3) which satisfies boundary conditions (1.2).

The general form of the variational problem (1.1) is

$$
v\left[y_{1}, y_{2}, \ldots, y_{n}\right]=\int_{x_{0}}^{x_{1}} F\left(x, y_{1}, y_{2}, \ldots, y_{n}, y_{1}^{\prime}, y_{2}^{\prime}, \ldots, y_{n}^{\prime}\right) d x,
$$

with the given boundary conditions for all functions:

$$
\begin{array}{ll}
y_{1}\left(x_{0}\right)=\alpha_{1}, & y_{2}\left(x_{0}\right)=\alpha_{2}, \ldots, y_{n}\left(x_{0}\right)=\alpha_{n}, \\
y_{1}\left(x_{1}\right)=\beta_{1}, & y_{2}\left(x_{1}\right)=\beta_{2}, \ldots, y_{n}\left(x_{1}\right)=\beta_{n} .
\end{array}
$$


Here the necessary condition for the extremum of the functional (1.4) is to satisfy the following system of second-order differential equations:

$$
F_{y_{i}}-\frac{d}{d x} F_{y_{i}^{\prime}}=0, \quad i=1,2, \ldots, n
$$

with boundary conditions given in (1.5). In the present work, we find the solution of variational problem by applying Adomian decomposition method on the Euler-Lagrange equations.

Also it is possible to define the variational problem for functionals dependent on higher-order derivatives in the following form [16]:

$$
v[y(x)]=\int_{x_{0}}^{x_{1}} F\left(x, y(x), y^{\prime}(x), \ldots, y^{(n)}(x)\right) d x,
$$

with the given boundary conditions

$$
\begin{aligned}
& y\left(x_{0}\right)=\alpha_{0}, \quad y^{\prime}\left(x_{0}\right)=\alpha_{1}, \ldots, y^{(n-1)}\left(x_{0}\right)=\alpha_{n-1}, \\
& y\left(x_{1}\right)=\beta_{0}, \quad y^{\prime}\left(x_{1}\right)=\beta_{1}, \ldots, y^{(n-1)}\left(x_{1}\right)=\beta_{n-1} .
\end{aligned}
$$

The function $y(x)$ which extermizes the functional (1.7) must satisfy the Euler-Poisson equation

$$
F_{y}-\frac{d}{d x} F_{y^{\prime}}+\frac{d^{2}}{d x^{2}} F_{y^{\prime \prime}}+\cdots+(-1)^{n} \frac{d^{n}}{d x^{n}} F_{y^{n}}=0,
$$

which is an ordinary differential equation of order $2 n$, with boundary conditions given in (1.8).

The Adomian decomposition method is useful for obtaining both a closed form and the explicit solution and numerical approximations of linear or nonlinear differential equations, and it is also quite straightforward to write computer codes. This method has been applied to obtain formal solution to a wide class of stochastic and deterministic problems in science and engineering involving algebraic, differential, integrodifferential, differential delay, integral and partial differential equations.

Generally this method is useful for problems that can be written in the following form which appears in the large number of problems in applied sciences:

$$
u-\Theta(u)=g
$$

where $u$ is unknown, $\Theta$ usually is a nonlinear operator, and $g$ is given. Depending on the nonlinear form $\Theta$, we can consider the Adomian decomposition method as an efficient method.

This method has been proposed by the American mathematician G. Adomian (19231996). It is based on the search for a solution in the form of a series and on decomposing the nonlinear operator into a series in which the terms are calculated recursively using Adomian polynomials [6]. 
The decomposition method was proven by many authors to be reliable and promising. It can be used for all types of differential equations, linear or nonlinear, homogeneous or inhomogeneous $[1-3,5,6]$. The technique has many advantages over the classical techniques, it avoids perturbation in order to find solutions of given nonlinear equations. The decomposition approach was used to handle a variety of linear and nonlinear problems and provides an immediate and convergent solution without any need for linearization or discretization.

In recent years a lot of attention has been developed to the study of the Adomian decomposition method to investigate various scientific models. This method is applied to solve various kinds of ordinary differential equations. Specially this method is useful for nonlinear differential equations [7]. Furthermore this method is used for finding the numerical solution of higher-order differential equations in [26-28].

The Adomian decomposition method which accurately computes the series solution is of great interest to applied science, engineering, physics, biology, and so forth. The method provides the solution in a rapidly convergent series with components that can be elegantly computed $[8,12-14,23]$. The present work is aimed at producing approximate solutions which are obtained in rapidly convergent series with elegantly computable components by the Adomian decomposition technique. It is well known in the literature that the decomposition method provides the solution in a rapidly convergent series where the series may lead to the solution in a closed form if it exists. The rapid convergence of the solution is guaranteed by the work conducted by Cherruault [11].

The organization of the rest of this paper is as follows. In Section 2, we apply the Adomian decomposition method on some ordinary differential equations with given suitable boundary conditions which arise from problems of calculus of variations. To present a clear overview of the method, we select several examples with analytical solutions in Section 3. A conclusion is presented in Section 4.

\section{Solution using the Adomian decomposition method}

Consider the Euler-Lagrange equation (1.3) in an operator form:

$$
L(y)-N(y)=f
$$

for $x_{0} \leq x \leq x_{1}$ where $L=d^{2} / d x^{2}$ is the second-order derivative operator, $N$ usually is a nonlinear operator which contains differential operators with order less than two, and $f$ is a given function. Assume that the inverse operator $L^{-1}$ exists and it can conveniently be taken as the definite integral for a function $h(x)$ in the following form:

$$
L^{-1}(h(x))=\int_{x_{0}}^{x} \int_{x_{0}}^{t_{2}} h\left(t_{1}\right) d t_{1} d t_{2}
$$

Applying the inverse operator $L^{-1}$ to both sides of (2.1) yields

$$
L^{-1} L(y)=L^{-1} N(y)+L^{-1} f .
$$


Thus we have

$$
y(x)-y\left(x_{0}\right)-y^{\prime}\left(x_{0}\right) x+y^{\prime}\left(x_{0}\right) x_{0}=L^{-1} N(y)+L^{-1} f
$$

or equivalently

$$
y(x)=\alpha+A x-A x_{0}+L^{-1} f+L^{-1} N(y)
$$

where $A=y^{\prime}\left(x_{0}\right)$. Now according to the decomposition procedure of Adomian, we construct the unknown function $y(x)$ by a sum of components defined by the following decomposition series:

$$
y(x)=\sum_{n=0}^{\infty} y_{n}(x)
$$

Based on the Adomian decomposition method, we consider the solution of (2.1) as the series (2.6) and take the nonlinear expressions $N(y)$ by the infinite series of the Adomian polynomials given by

$$
N(y)=\sum_{n=0}^{\infty} N_{n}
$$

where components $N_{n}$ are appropriate Adomian's polynomials which are calculated using methods introduced in [6]. Adomian polynomials are found for calculating the nonlinear operator $N_{n}$ in the following form:

$$
N_{n}\left(y_{0}, y_{1}, \ldots, y_{n}\right)=\frac{1}{n !} \frac{d^{n}}{d \lambda^{n}}\left[N\left[\sum_{k=0}^{\infty} \lambda^{k} y_{k}\right]\right]_{\lambda=0}, \quad n \geq 0 .
$$

This formula is calculated in computer code easily. Other general formulas of Adomian polynomials can be found in $[4,25]$. Notice that if $N$ be a linear operator then we have $N_{n}=y_{n}$.

Now by the decomposition method of Adomian we have the following recursive relations:

$$
y_{0}(x)=\alpha+A x-A x_{0}+L^{-1} f(x), \quad y_{n+1}(x)=L^{-1} N_{n}, \quad n \geq 0 .
$$

The resulted solution converges [11] to the closed form solution if an exact solution exists for the Euler-Lagrange equation. The most important work about convergence has been carried by Cherruault [11]. Other references about theoretical treatments of the convergence of Adomian decomposition method are found in [3]. In [3, 24] some results are obtained about the improvement of this method that let us solve linear and nonlinear equations. A new approach of convergence of the decomposition method has been presented in Ngarhasta et al. [20]. 
6 Application of Adomian's method in calculus of variations

By calculating the terms $y_{0}, y_{1}, y_{2}, \ldots$, the solution $y$ of the Euler-Lagrange equation (1.3) can be obtained upon substituting the resulting terms in (2.6).

Based on the Adomian decomposition method, we constructed the solution $y$ as

$$
y=\lim _{n \rightarrow \infty} \phi_{n}
$$

where the $(n+1)$-term approximation of the solution is defined in the following form:

$$
\phi_{n}=\sum_{k=0}^{n} y_{k}(x), \quad n \geq 0 .
$$

The solution here is given in a series form that generally converges very rapidly in real physical problems.

Applying the decomposition procedure of Adomian, we find that the series solution of $y(x)$ follows with a constant $A$ which is unknown. To find this constant we impose the boundary condition at $x=x_{1}$ to the obtained approximation of the solution defined in (2.11) which results in an equation in $A$. By solving this equation that usually is nonlinear, we find $A$ and then the solution of the Euler-Lagrange equation follows immediately.

We can apply the above scheme on the second-order system of ordinary differential equations (1.6) and find a solution for this equation. In the operator form we have

$$
L\left(y_{i}\right)-N_{i}\left(y_{1}, y_{2}, \ldots, y_{n}\right)=f_{i}, \quad i=1,2, \ldots, n,
$$

where $x_{0} \leq x \leq x_{1}, N_{i}, i=1, \ldots, n$, are nonlinear operators which contain differential operators with order less than two, and $f_{i}, i=1, \ldots, n$, are given functions.

Thus we have

$$
y_{i}(x)=\alpha_{i}+A_{i} x-A_{i} x_{0}+L^{-1} f_{i}+L^{-1} N_{i}\left(y_{1}, y_{2}, \ldots, y_{n}\right)
$$

where $A_{i}=y_{i}^{\prime}\left(x_{0}\right)$. By the Adomian decomposition method we have the following recursive relations:

$$
y_{i 0}(x)=\alpha_{i}+A_{i} x-A_{i} x_{0}+L^{-1} f_{i}(x), \quad y_{i(n+1)}(x)=L^{-1} N_{i n}, \quad n \geq 0,
$$

and the series solutions are given in the following form:

$$
y_{i}(x)=\sum_{n=0}^{\infty} y_{i n}(x) .
$$


Series solutions obtained in (2.15) are followed by constants $A_{i}, i=1, \ldots, n$. By imposing the boundary conditions at $x=x_{1}$ to the approximations of the series solutions

$$
\phi_{\text {in }}=\sum_{k=0}^{n} y_{i k}(x), \quad n>0,
$$

we obtain an algebraic system in $A_{i}, i=1, \ldots, n$. This system usually is nonlinear. Thus finding the solution of this system using analytical methods is not easy. Here we use the well-known Newton method with an appropriate initial point to find the solution of this system numerically.

Similarly if we consider operator $L=d^{2 n} / d x^{2 n}$ and $N$ to be a nonlinear operator which contains differential operators with order less than $2 n$, the above procedure can be repeated to find the solution of the problem (1.9).

A reliable modification of the Adomian decomposition method has been developed in [24]. In this approach $y_{0}$ is considered to contain minimal number of terms. This work has a considerable effect on facilitating the formulation of the Adomian polynomials $A_{n}$.

In the next section some examples are used to validate the proposed method.

\section{Test examples}

To show the efficiency of the new method described in the previous section, we present some examples. These examples are chosen such that there exist analytical solutions for them to give an obvious overview of the Adomian decomposition method.

Example 3.1. Consider the following variational problem:

$$
\min v=\int_{0}^{1}\left(y(x)+y^{\prime}(x)-4 \exp (3 x)\right)^{2} d x
$$

with given boundary conditions

$$
y(0)=1, \quad y(1)=e^{3} .
$$

The corresponding Euler-Lagrange equation is

$$
y^{\prime \prime}-y-8 \exp (3 x)=0
$$

with boundary conditions (3.2). The exact solution of this problem is $y(x)=\exp (3 x)$. Using the operator form of (3.3) we have

$$
N y=y+8 \exp (3 x)
$$

Thus

$$
y(x)=1+A x+L^{-1}(8 \exp (3 x))+L^{-1}(y(x)) .
$$


8 Application of Adomian's method in calculus of variations

Now the decomposition procedure of Adomian results in

$$
\begin{gathered}
\sum_{k=0}^{\infty} y_{k}(x)=1+A x+L^{-1}(8 \exp (3 x))+L^{-1}\left(\sum_{k=0}^{\infty} y_{k}(x)\right), \\
y_{0}(x)=1+A x+L^{-1}(8 \exp (3 x))=\frac{1}{9}+A x+\frac{8}{9} \exp (3 x)-\frac{8}{3} x, \\
y_{1}(x)=L^{-1}\left(y_{0}(x)\right)=\frac{1}{18} x^{2}+\frac{1}{6} A x^{3}+\frac{8}{81} \exp (3 x)-\frac{4}{9} x^{3}-\frac{8}{27} x-\frac{8}{81}, \\
y_{2}(x)=L^{-1}\left(y_{1}(x)\right)=\frac{1}{216} x^{4}+\frac{1}{120} A x^{5}+\frac{8}{729} \exp (3 x)-\frac{1}{45} x^{5}-\frac{4}{81} x^{3}-\frac{4}{81} x^{2}-\frac{8}{243} x-\frac{8}{729},
\end{gathered}
$$

and so on. This gives the approximation of the $y(x)$ in a series form. Now to find the constant $A$, the boundary condition at $x=1$ is imposed on the $n$-term approximation $\phi_{n}$ in (2.11). For example for $n=4$ we obtain

$$
1.175201168 A=3.52560388
$$

which results in

$$
A=3.000000320 \text {. }
$$

By replacing $A$ in the $\phi_{4}$, an approximate solution is obtained for $y(x)$. Higher accuracy is obtained using more components of $y(x)$. Figure 3.1 shows the error of $\phi_{4}$. Furthermore we have

$$
v\left(\phi_{4}\right)-v(y)=0.5273450967 e-12 .
$$

It is clear that in this example the Adomian decomposition method can be considered as an efficient method.

Example 3.2. Consider the following brachistochrone problem [15]:

$$
\min v=\int_{0}^{1}\left[\frac{1+y^{\prime 2}(x)}{1-y(x)}\right]^{1 / 2} d x
$$

let the boundary conditions be

$$
y(0)=0, \quad y(1)=-0.5
$$

In this case the Euler-Lagrange equation is written in the following form:

$$
y^{\prime \prime}=-\frac{1}{2} \frac{1+y^{\prime 2}}{y-1} .
$$




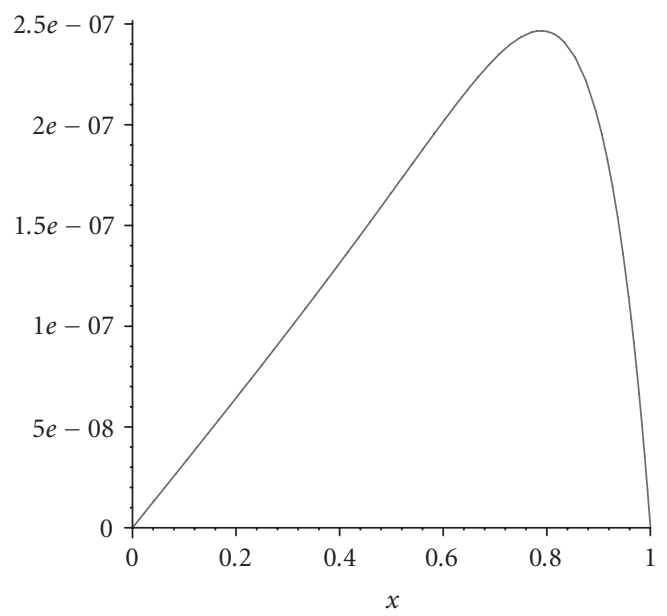

Figure 3.1. Error function $\phi_{4}-y(x)$ for $0 \leq x \leq 1$.

By imposing the boundary condition at $x=1$ on the four-term approximation

$$
\phi_{3}=\sum_{k=0}^{3} y_{k}
$$

and solving the resulted nonlinear equation, we obtain

$$
A=-0.78503193483611740425 \text {. }
$$

In this case we have

$$
v\left(\phi_{3}\right)-v(y)=0.2232 e-6
$$

which shows the high accuracy of the method for nonlinear problems. Notice that the direct methods for solving the variational problem provide an algebraic system of equations. Solving such equations is very time consuming. But as we saw, using the decomposition procedure of Adomian, the solution of the problem is obtained very fast without solving any algebraic system of equations.

Example 3.3. In this example we consider the following variational problem [16]:

$$
\min v=\int_{0}^{\pi / 2}\left(y^{\prime \prime} 2-y^{2}+x^{2}\right) d x
$$

that satisfies the conditions

$$
y(0)=1, \quad y^{\prime}(0)=0, \quad y\left(\frac{\pi}{2}\right)=0, \quad y^{\prime}\left(\frac{\pi}{2}\right)=-1 .
$$


The corresponding Euler-Poisson equation is

$$
y^{(4)}-y=0,
$$

or equivalently

$$
y(x)=1+\frac{1}{2 !} A x^{2}+\frac{1}{3 !} B x^{3}+L_{4}^{-1}(y(x)),
$$

where $L_{4}^{-1}$ is defined for a function $h(x)$ by

$$
L_{4}^{-1}(h(x))=\int_{0}^{x} \int_{0}^{t_{4}} \int_{0}^{t_{3}} \int_{0}^{t_{2}} h\left(t_{1}\right) d t_{1} d t_{2} d t_{3} d t_{4},
$$

in which $A=y^{\prime \prime}(0)$ and $B=y^{(3)}(0)$.

Using the decomposition method we have

$$
\begin{gathered}
y_{0}(x)=1+\frac{1}{2 !} A x^{2}+\frac{1}{3 !} B x^{3}, \\
y_{1}(x)=L_{4}^{-1}\left(y_{0}(x)\right)=\frac{1}{24} x^{4}+\frac{1}{720} A x^{6}+\frac{1}{5040} B x^{7},
\end{gathered}
$$

and so on. By imposing the boundary conditions at $x=1$ on $\phi_{4}$, we obtain the following linear equations:

$$
\begin{gathered}
1.254589239 A+0.6506494512 B=-1.254589239, \\
1.650649451 A+1.254589239 B=-1.650649450,
\end{gathered}
$$

which results in

$$
A=-1.000000001, \quad B=0.1254589241 e-8 .
$$

In Figure 3.2 the error function $\phi_{4}-y(x)$ is plotted. Furthermore, we have

$$
v\left(\phi_{4}\right)-v(y)=-0.4228 e-4 .
$$

Obviously a better approximation can be found using more components of $y(x)$.

\section{Conclusion}

Adomian decomposition method is used for finding the solution of the ordinary differential equations which arise from problems of calculus of variations. It is also important that the Adomian decomposition method does not require discretization of the variables. It is not affected by computation round errors and one is not faced with necessity of large computer memory and time. The decomposition approach is implemented directly in a straightforward manner without using restrictive assumptions or linearization. Comparing the results with other works, the Adomian decomposition method was clearly reliable if compared with the grid point techniques where the solution is defined at grid points only. It is important that this method unlike the most numerical techniques provides a closed form of the solution. 


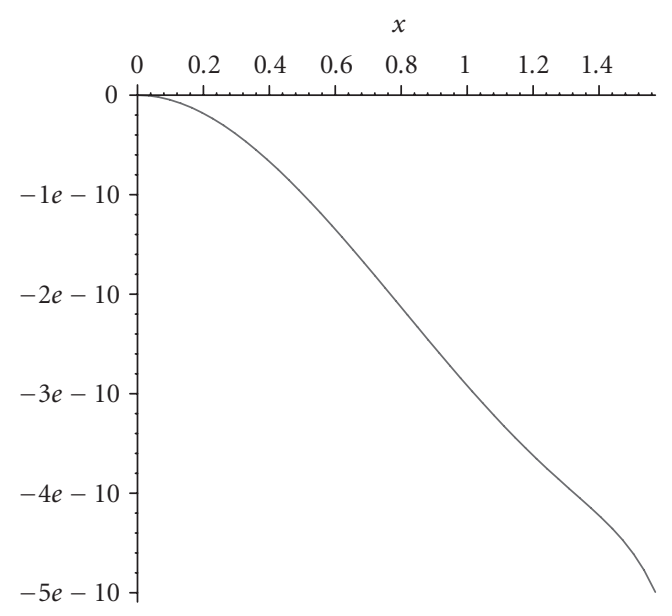

Figure 3.2. Error function $\phi_{4}-y(x)$ for $0 \leq x \leq \pi / 2$.

\section{Acknowledgment}

The authors would like to thank the anonymous referees for carefully reading the paper.

\section{References}

[1] G. Adomian, A new approach to the heat equation-an application of the decomposition method, Journal of Mathematical Analysis and Applications 113 (1986), no. 1, 202-209.

[2] Modification of the decomposition approach to the heat equation, Journal of Mathematical Analysis and Applications 124 (1987), no. 1, 290-291.

[3] _ A review of the decomposition method in applied mathematics, Journal of Mathematical Analysis and Applications 135 (1988), no. 2, 501-544.

[4] _ Nonlinear Stochastic Systems Theory and Applications to Physics, Mathematics and Its Applications, vol. 46, Kluwer Academic, Dordrecht, 1989.

[5] __ Solving frontier problems modelled by nonlinear partial differential equations, Computers \& Mathematics with Applications 22 (1991), no. 8, 91-94.

[6] Solving Frontier Problems of Physics: The Decomposition Method, Fundamental Theories of Physics, vol. 60, Kluwer Academic, Dordrecht, 1994.

[7] G. Adomian and R. Rach, Solution of nonlinear ordinary and partial differential equations of physics, Journal of Mathematical and Physical Sciences 25 (1991), no. 5-6, 703-718 (1993).

[8] E. Babolian, J. Biazar, and A. R. Vahidi, The decomposition method applied to systems of Fredholm integral equations of the second kind, Applied Mathematics and Computation 148 (2004), no. 2, $443-452$.

[9] R. Y. Chang and M. L. Wang, Shifted Legendre direct method for variational problems, Journal of Optimization Theory and Applications 39 (1983), no. 2, 299-307.

[10] C. F. Chen and C. H. Hsiao, A Walsh series direct method for solving variational problems, Journal of the Franklin Institute 300 (1975), no. 4, 265-280.

[11] Y. Cherruault, Convergence of Adomian's method, Mathematical and Computer Modelling 14 (1990), 83-86. 
[12] M. Dehghan, Application of the Adomian decomposition method for two-dimensional parabolic equation subject to nonstandard boundary specifications, Applied Mathematics and Computation 157 (2004), no. 2, 549-560.

[13] - The solution of a nonclassic problem for one-dimensional hyperbolic equation using the decomposition procedure, International Journal of Computer Mathematics 81 (2004), no. 8, 979989.

[14] - The use of Adomian decomposition method for solving the one-dimensional parabolic equation with non-local boundary specifications, International Journal of Computer Mathematics 81 (2004), no. 1, 25-34.

[15] P. Dyer and S. R. McReynolds, The Computation and Theory of Optimal Control, Mathematics in Science and Engineering, vol. 65, Academic Press, New York, 1970.

[16] L. Elsgolts, Differential Equations and the Calculus of Variations, Mir, Moscow, 1977, translated from the Russian by G. Yankovsky.

[17] I. M. Gelfand and S. V. Fomin, Calculus of Variations, revised English edition translated and edited by R. A. Silverman, Prentice-Hall, New Jersey, 1963.

[18] I. R. Horng and J. H. Chou, Shifted Chebyshev direct method for solving variational problems, International Journal of Systems Science 16 (1985), no. 7, 855-861.

[19] C. Hwang and Y. P. Shih, Laguerre series direct method for variational problems, Journal of Optimization Theory and Applications 39 (1983), no. 1, 143-149.

[20] N. Ngarhasta, B. Some, K. Abbaoui, and Y. Cherruault, New numerical study of Adomian method applied to a diffusion model, Kybernetes 31 (2002), no. 1, 61-75.

[21] M. Razzaghi and M. Razzaghi, Fourier series direct method for variational problems, International Journal of Control 48 (1988), no. 3, 887-895.

[22] _ Instabilities in the solution of a heat conduction problem using Taylor series and alternative approaches, Journal of the Franklin Institute 326 (1989), no. 5, 683-690.

[23] M. Tatari and M. Dehghan, Numerical solution of Laplace equation in a disk using the Adomian decomposition method, Physica Scripta 72 (2005), no. 5, 345.

[24] A.-M. Wazwaz, A reliable modification of Adomian decomposition method, Applied Mathematics and Computation 102 (1999), no. 1, 77-86.

[25] _ A new algorithm for calculating Adomian polynomials for nonlinear operators, Applied Mathematics and Computation 111 (2000), no. 1, 53-69.

[26] _ Approximate solutions to boundary value problems of higher order by the modified decomposition method, Computers \& Mathematics with Applications 40 (2000), no. 6-7, 679-691.

[27] , The numerical solution of fifth-order boundary value problems by the decomposition method, Journal of Computational and Applied Mathematics 136 (2001), no. 1-2, 259-270.

[28] _ The numerical solution of sixth-order boundary value problems by the modified decomposition method, Applied Mathematics and Computation 118 (2001), no. 2-3, 311-325.

Mehdi Dehghan: Department of Applied Mathematics, Faculty of Mathematics and Computer Science, Amirkabir University of Technology, 424 Hafez Avenue,

Tehran 15875-4413, Iran

E-mail address: mdehghan@aut.ac.ir

Mehdi Tatari: Department of Applied Mathematics, Faculty of Mathematics and Computer Science, Amirkabir University of Technology, 424 Hafez Avenue,

Tehran 15875-4413, Iran

E-mail address: mehditatari@aut.ac.ir 


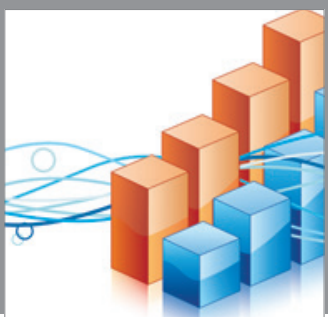

Advances in

Operations Research

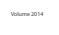

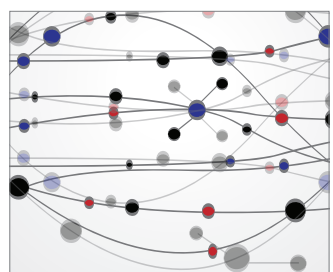

\section{The Scientific} World Journal
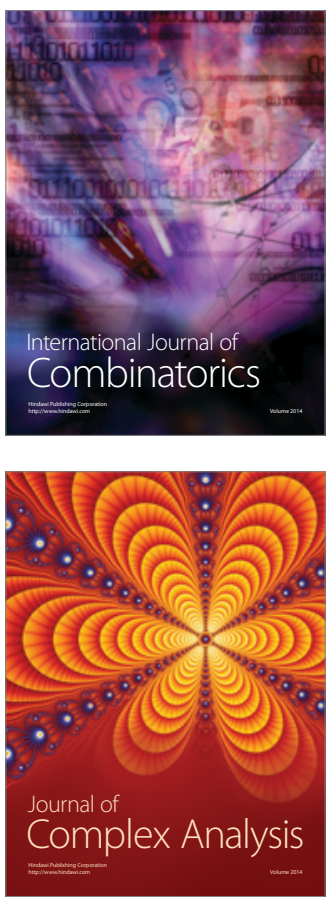

International Journal of

Mathematics and

Mathematical

Sciences
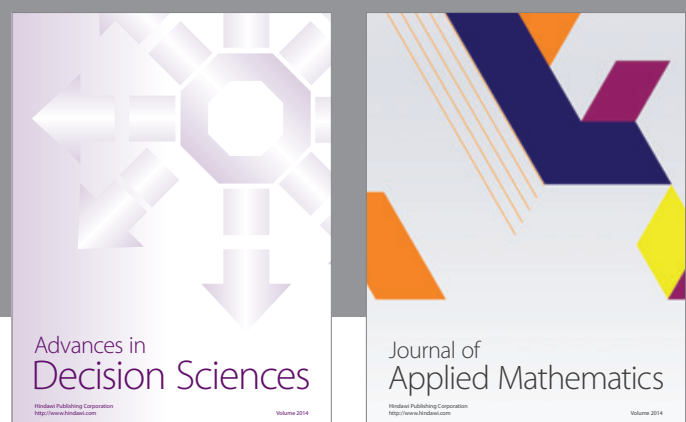

Journal of

Applied Mathematics
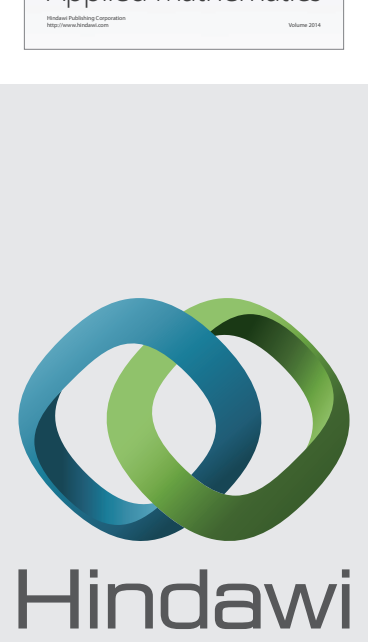

Submit your manuscripts at http://www.hindawi.com
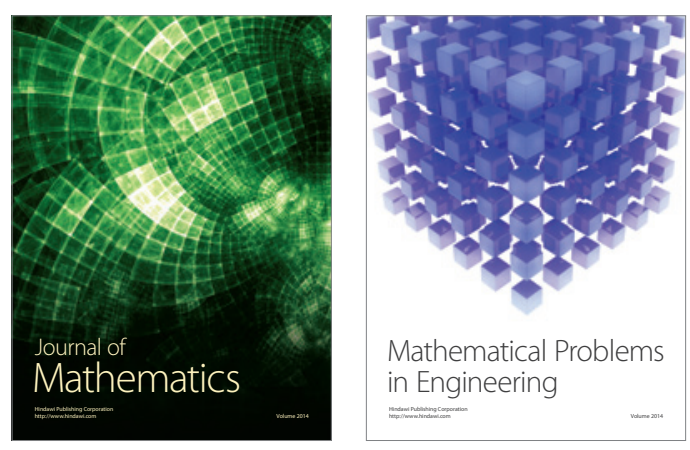

Mathematical Problems in Engineering
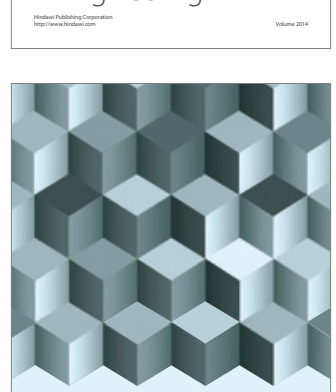

Journal of

Function Spaces
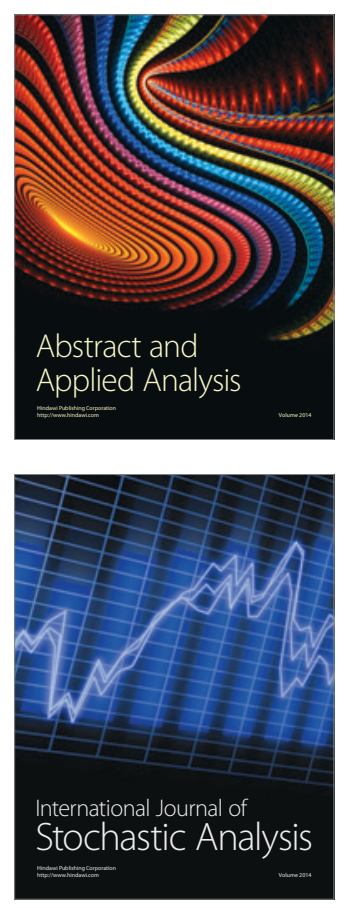

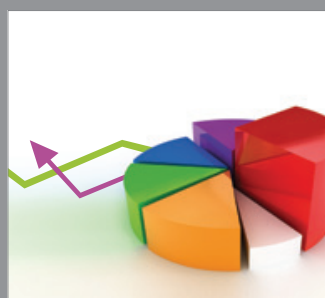

ournal of

Probability and Statistics

Promensencen
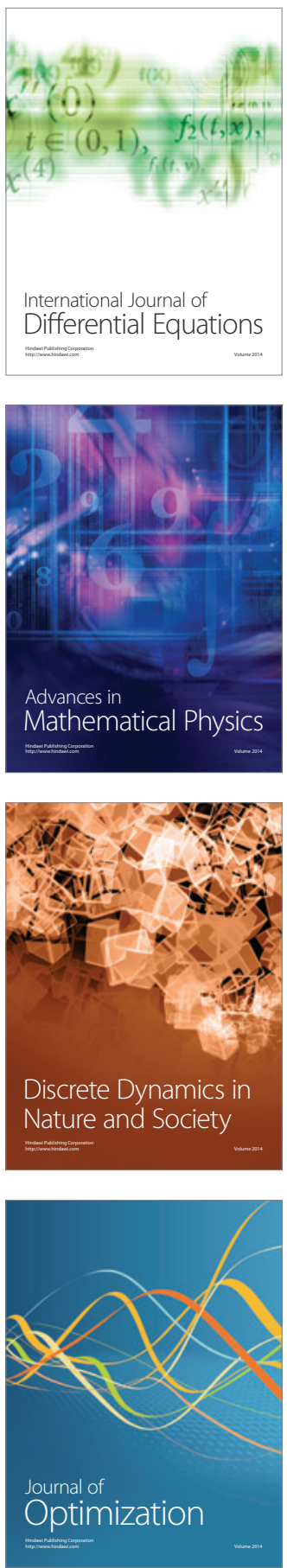